Ann. Sci. forest., 1968, 25 (1), 3-23.

\title{
TESTS PRÉCOCES DE LA QUALITÉ DU BOIS SUR 25 PROVENANGES D'ABIES GRANDIS
}

\author{
H. POLGE \\ Station de Recherches sur la Qualité des Bois, \\ Centre national de Recherches forestières, 54 - Nancy \\ Institut national de la Recherche agronomique
}

SOMMAIRE

Des déterminations de densité des tiges, de densité des branches, de largeur et de composition d'un accroissement annuel ont été faites pour 25 provenances d'Abies grandis.

La méthodologie utilisée, compte tenu de la taille très réduite des échantillons, est décrite, puis sont exposés les résultats de nombreuses études de corrélation faites entre ces diverses données et d'autres caractéristiques se rapportant à la croissance des plants. Un test de comparaison de moyennes fait ressortir l'intérêt du pourcentage de bois d'été pour différencier les provenances.

\section{INTRODUCTION}

Dans le dernier numéro de ces Annales, LACAzE a tiré les premières conclusions, au stade pépinière, d'une expérimentation sur diverses provenances d'Abies grandis, d'un triple point de vue morphologique, phénologique et auxométrique.

Les résultats dont il est fait état ont été obtenus dans 4 dispositifs expérimentaux différents installés par ses soins, 3 dans la pépinière d'Amance, et le quatrième dans celle de Velaine.

Parallèlement à ces investigations, certaines études de caractère technologique ont été entreprises par la Station de Recherches sur la Qualité des Bois dans une répétition d'un des dispositifs (le n ${ }^{0} \mathrm{P}$ 031-1) ; il s'agit là d'un travail qui n'est pas pleinement satisfaisant pour de multiples raisons : erreurs de manipulation, échantillonnage insuffisant, impossibilité de rassembler les résultats avec ceux obtenus par la Station d'Amélioration dans une étude statistique commune.

Cependant, malgré ces imperfections, quelques observations intéressantes ont pu être faites à cette occasion, et il peut ne pas être inutile d'en rendre compte ici car c'est, semble-t-il, la première fois que des renseignements de cette nature sont obtenus sur des échantillons d'aussi faibles dimensions, et de plus, certaines des informations ainsi recueillies étaient a priori tout a fait inattendues. 


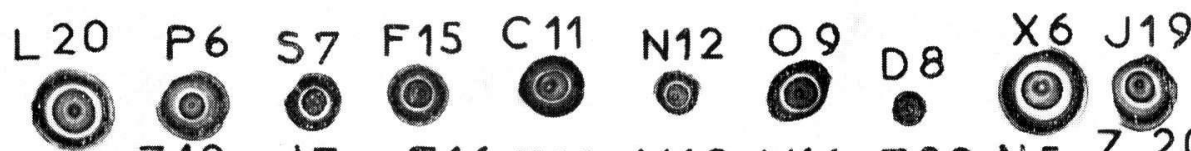
P3 Z12 J7 F16 E10 M18 V16 T20 N5 Z 20 () () (ㅇ) () () ()

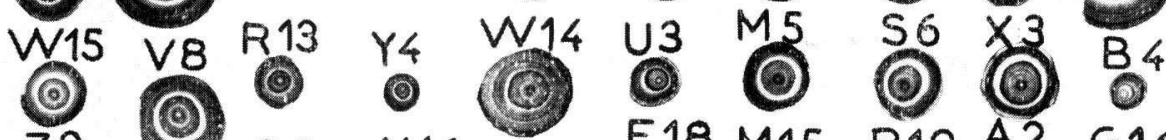
$22 N C_{2} \times 16$ R10 E18 M15 B10 A2 G16

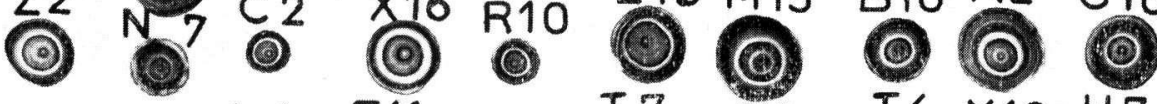
H16 V8 L6 E11 114 (2) G3 T4 X19 H7

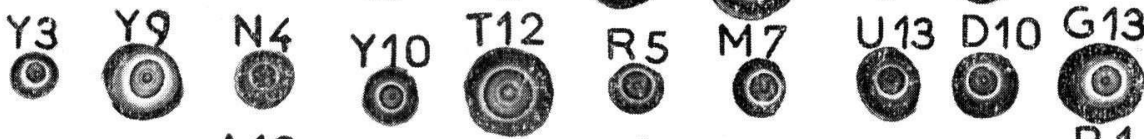
A7 Q9 119 F19 P17 H20 F3 51016 B1

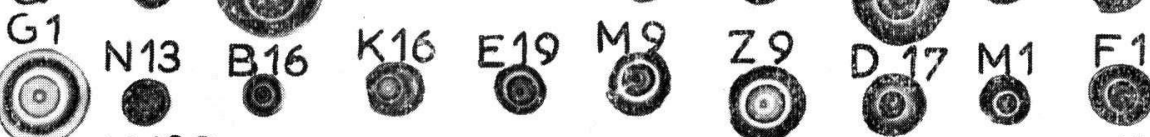

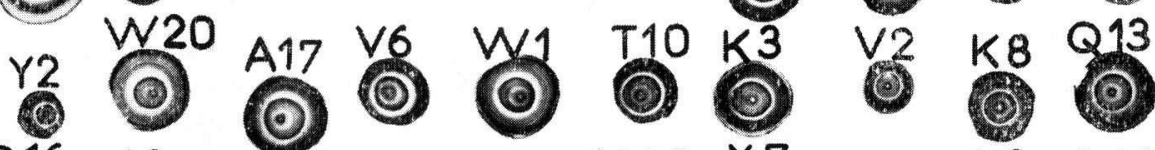
D16 J9 Q5 O13 N16 W5 Y7 N9 A3 R19 P19 K19 E15 E4 C16 E17 L2 $\times 18 \mathrm{H}^{\mathrm{H}} \times 2$ $415 \times 145150619$ U15 k14 S15 06 u11 P7 R16 Q10 Q19 G19

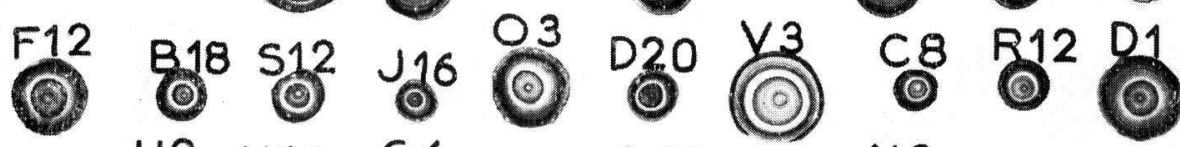

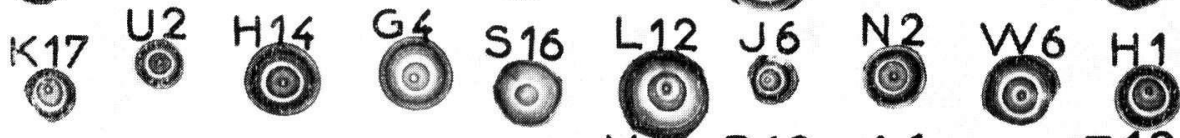

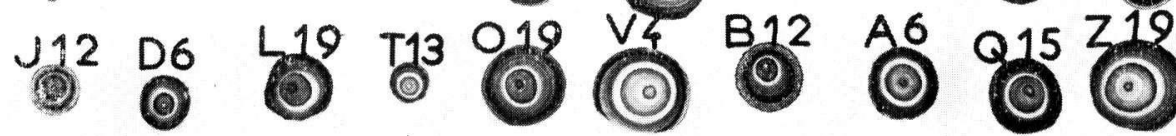




\section{1. - MATÉRIEL ET MÉTHODE}

Les déterminations suivantes ont été faites sur plants de 3 ans reçus au cours de l'hiver 19641965 : poids anhydre de la partie aérienne, infra-densité tiges, infra-densité branches, largeur du cerne 1964, largeur bois de printemps 1964, largeur bois d'été 1964, pourcentage de bois d'été 1964.

L'échantillonnage comprenait 20 plants pour chaque provenance, à l'exception de Leaburg I, Palmer, Clarkia et Leaburg II qui n'en comportaient que 19. De plus, lors de la détermination des densités, la totalité des échantillons de la provenance Leaburg I et 6 échantillons de la provenance Lewis ont été détruits accidentellement par surchauffe de l'étuve; la densité du bois n'a donc pas pu être calculée sur la première, et l'a été sur un échantillonnage réduit à 14 individus pour la seconde. Enfin, les plants étudiés pour la provenance Nanaïmo n'ont, par erreur, pas été extraits dans la même répétition que l'ensemble des autres, et les résultats la concernant sont par suite donnés sous toutes réserves.

Les échantillons utilisés pour les calculs de densité consistaient en fragments de tige de $2,5 \mathrm{~cm}$ de longueur environ prélevés au-dessus du collet, empâtement déduit; en ce qui concerne les branches, des tronçons de 1 à $2 \mathrm{~cm}$ de longueur ont été découpés dans chacune des branches principales du premier verticille, et ont servi aux calculs de densité qui ont été faits sur l'ensemble de ces fragments, et non pas sur chacun d'eux pris isolément.

Les densités du bois des tiges ont été calculées à partir des volumes saturés mesurés au voluménomètre à mercure; pour le bois des branches par contre, la méthode employée a été celle dite « de la saturation intégrale » déjà décrite par ailleurs (Polge H., 1963. Contribution à l'étude de la qualité du bois des principales essences résineuses exotiques utilisées dans les reboisements français. Ann. Ec. for. Nancy, tome XX, fascicule 3); les valeurs données sont donc théoriquement, dans tous les cas, des valeurs d'infra-densité, ou encore de densité basale (rapport du poids anhydre au volume saturé).

La mise à jour des résultats a été retardée par les nombreux problèmes posés par les faibles dimensions des échantillons et par le fait que le fil du bois correspondait à leur grande longueur; 4 et parfois 5 déterminations successives de la densité ont été faites sur les tiges, les premiers calculs ayant été faussés par la présence d'écorce ou par une saturation insuffisante; les chiffres fournis pour les tiges ne concernent que le bois seul, les échantillons ayant été entièrement décortiqués ; par contre, l'enlèvement de l'écorce s'est avérée impossible sur les branches, dont certaines n'atteignaient pas un millimètre de diamètre, en sorte que les valeurs moyennes indiquées le sont sous toutes réserves, et ne doivent pas être comparées globalement à celles trouvées pour les tiges; cependant, tous les échantillons ayant subi simultanément les mêmes traitements, les chiffres demeurent valables pour classer entre elles les diverses provenances.

Il convient de mentionner en outre qu'un grand nombre de plants comportaient des zones étendues de bois de compression, représentant parfois une fraction importante de la surface en section transversale et ayant pu fausser quelque peu les valeurs de densité trouvées; fort heureusement, ce bois de compression semble se manifester avec une ampleur comparable dans les diverses provenances, et paraît notamment ne pas être lié à la vigueur de croissance.

L'étude des largeurs de cernes et des largeurs respectives du bois de printemps et du bois d'été sur un aussi grand nombre d'échantillons de très faibles dimensions a été elle aussi très délicate, et de nombreux tâtonnements ont été nécessaires pour mettre au point la méthode qui a été finalement retenue, et dont les principes sont les suivants : des rondelles de tige de 2,1 mm d'épaisseur prélevées à la partie inférieure des échantillons, ont été radiographiées ensemble, après avoir été disposées de façon aléatoire sur deux films de format $18 \times 24 \mathrm{~cm}$; chaque image radiographique a été examinée au microscope de projection, et on a mesuré, au grossissement 20 , la largeur totale du cerne 1964 et celle du bois d'été, ou bois final (donnant par différence la largeur du bois de printemps, ou bois initial), le tout sur deux rayons de direction perpendiculaire à celle de l'excentricité maximale occasionnée par le bois de compression; dans les cas douteux, il a été fait appel aux méthodes de l'analyse densitométrique pour préciser la limite bois initial-bois final.

La figure 1 représente un positif grandeur nature d'une portion de l'une des radiographies; on peut y observer la taille extrêmement réduite de certains échantillons, ainsi qu'une très grande variabilité inter et intra-provenances à tous points de vue : diamètre de la tige, épaisseur et densité propre des divers cernes ou parties de cernes, épaisseur et densité propre de l'écorce. En outre sont regroupées, à titre d'exemple, sur les figures 2 et 2 bis, les images, prises suivant un rayon, de deux rondelles caractéristiques, l'une à fort, l'autre à faible pourcentage de bois d'été, et les courbes de variation de densité correspondantes, le tout au grossissement 20.

Les calculs statistiques dont il est rendu compte ci-dessous ont dû être faits à la Station de Recherches sur la Qualité des Bois ; ce sont des calculs sommaires qui ne portent en fait, sauf exception, que 
H. POLGE

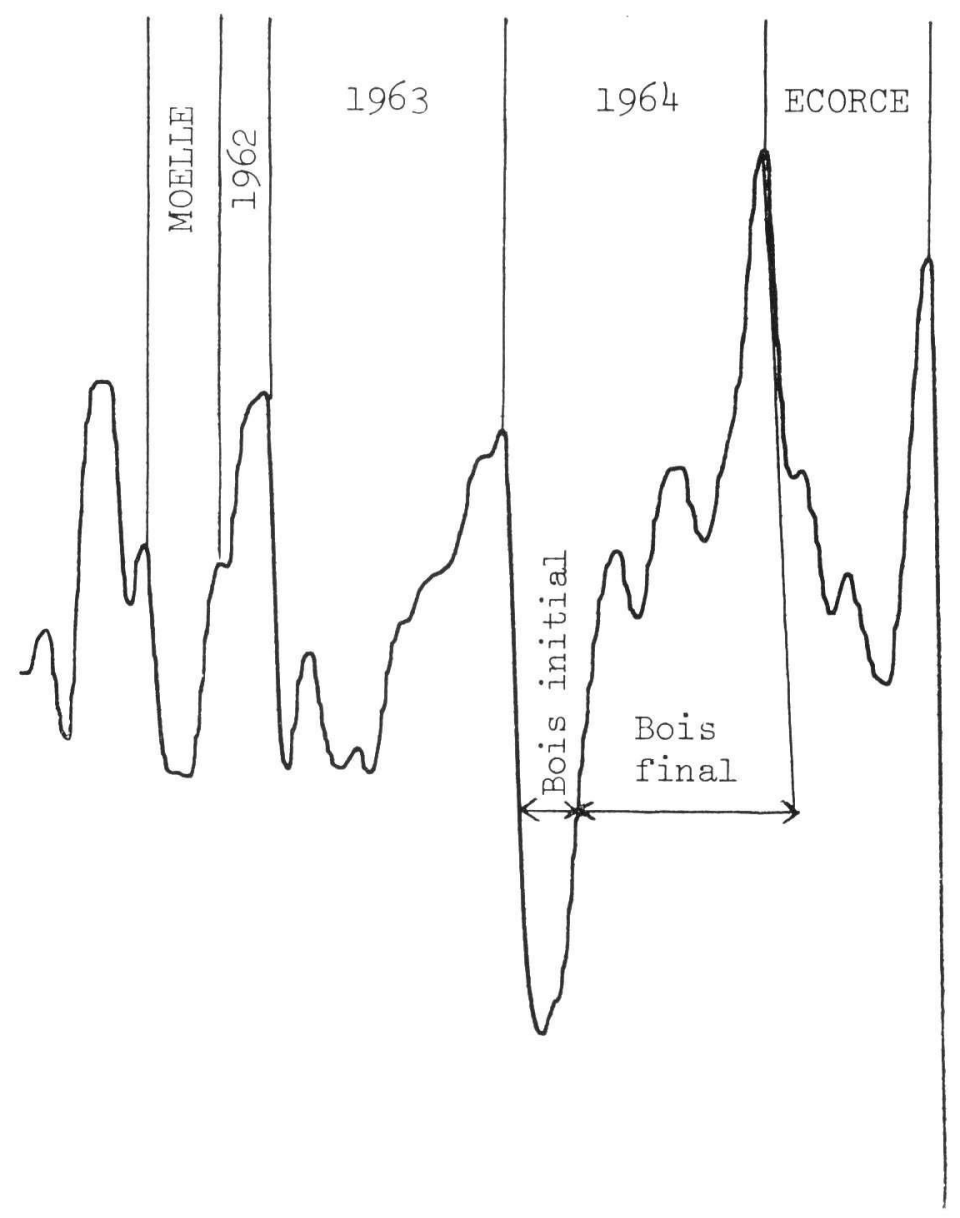

FIG. 2. - Positif de radiographie, agrandie 20 fois, et profil densitométrique pour un échantillon à forte texture.

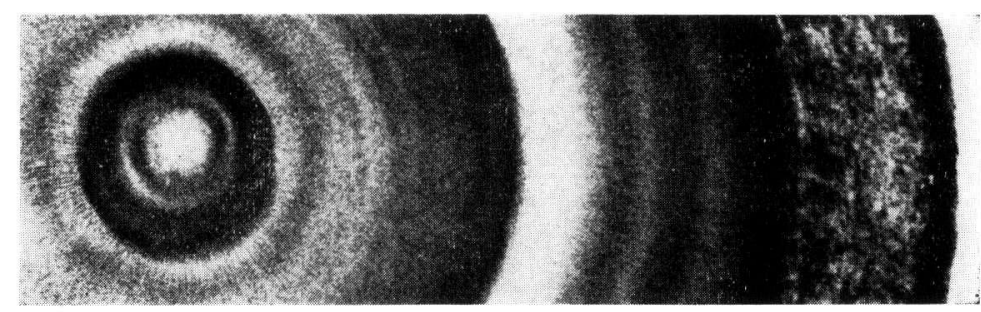

Échantillon Prospect $n^{0} 12$

Pourcentage bois final : $77 \%$ 
TESTS PRÉCOCES DE LA QUALITÉ DU BOIS

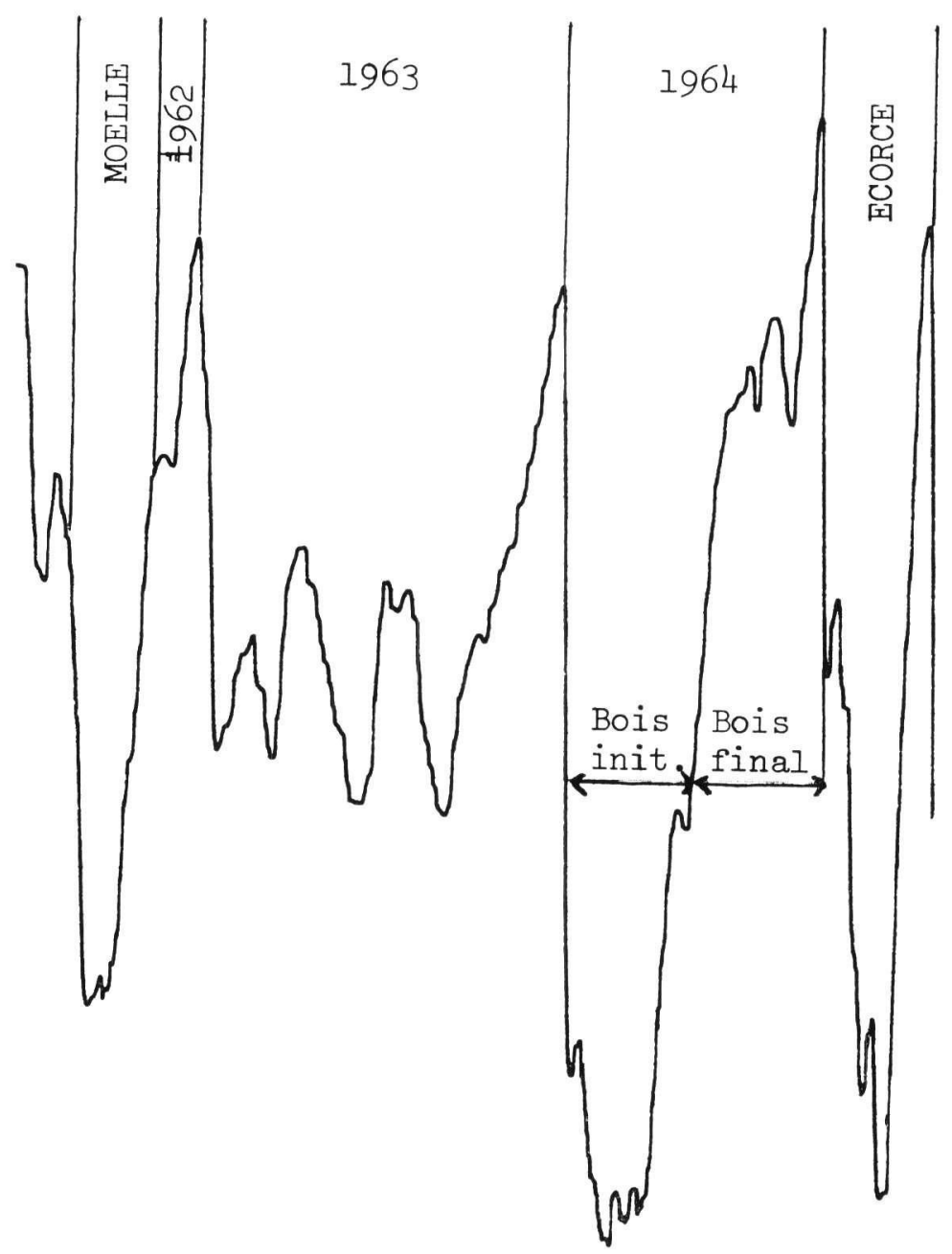

FIG. 2 bis. - Positif de radiographie, agrandie 20 fois, et profil densitométrique pour un échantillon à faible texture.

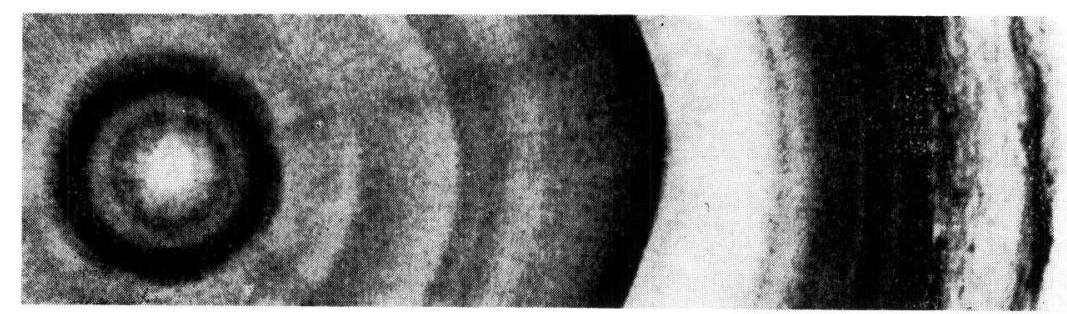

Échantillon Les Barres-L $n^{0} 3$

Pourcentage bois final : $50 \%$ 
sur des moyennes par provenance; l'ensemble des 6280 données recueillies a cependant été conservé et peut être fourni sur simple demande.

Sur les diverses figures, des points représentatifs différents ont été utilisés, suivant les zones d'origine géographique des provenances :

- un simple point pour les provenances cascadiennes,

- un cercle pour les provenances continentales,

- un carré pour les provenances artificielles,

- une croix ordinaire pour les provenances côtières,

- une croix de Saint-André pour la provenance Serandon, qui est supposée correspondre à un hybride d'Abies concolor.

\section{2. - RÉSULTATS}

\subsection{Résultats d'ensemble}

Ils sont consignés dans le tableau suivant :

TABLEAU 1

\begin{tabular}{|c|c|c|c|c|c|c|c|}
\hline Provenance & $\begin{array}{l}\text { Poids } \\
\text { anhydre } \\
\text { moyen } \\
\text { partie } \\
\text { aérienne } \\
\text { en g }\end{array}$ & $\begin{array}{l}\text { Infra- } \\
\text { densité } \\
\text { moyenne } \\
\text { tige } \\
\mathrm{g} / \mathrm{dm}^{3}\end{array}$ & $\begin{array}{l}\text { Infra- } \\
\text { densité } \\
\text { moyenne } \\
\text { branche } \\
\mathrm{g} / \mathrm{dm}^{3}\end{array}$ & $\begin{array}{c}\text { Largeur } \\
\text { moyenne } \\
\text { cerne } \\
1964 \\
\mathrm{~mm} / 100\end{array}$ & $\begin{array}{c}\text { Largeur } \\
\text { moyenne } \\
\text { bois de } \\
\text { prin- } \\
\text { temps } \\
\mathrm{mm} / 100\end{array}$ & $\begin{array}{l}\text { Largeur } \\
\text { moyenne } \\
\text { bois } \\
\text { d'été } \\
\mathrm{mm} / 100\end{array}$ & $\begin{array}{l}\text { Pourcen- } \\
\text { tage } \\
\text { moyen } \\
\text { bois d'été }\end{array}$ \\
\hline PE-ELL $\ldots \ldots \ldots$ & 21,4 & 529 & 429 & 146 & 32 & 114 & 78 \\
\hline LEWIS $\ldots \ldots \ldots \ldots$ & 11,7 & 538 & 395 & 119 & 24 & 95 & 80 \\
\hline LEABURG I $\ldots \ldots$ & 9,4 & & & 96 & 20 & & \\
\hline $\begin{array}{l}\text { CAMPBELL } \\
\text { RIVER } \ldots \ldots \ldots \ldots\end{array}$ & 13.1 & & 446 & 123 & & & \\
\hline PEZANIN $\ldots \ldots$ & 17,6 & 542 & 441 & $\begin{array}{l}123 \\
129\end{array}$ & $\begin{array}{l}25 \\
31\end{array}$ & $\begin{array}{l}98 \\
98\end{array}$ & $\begin{array}{l}80 \\
76\end{array}$ \\
\hline SNOQUALMIE & & & & & & & \\
\hline PASS $\ldots \ldots \ldots$ & 12,0 & 529 & 423 & 105 & 25 & 80 & 76 \\
\hline SERANDON $\ldots$. & 9,3 & 509 & 376 & 115 & 28 & 87 & 76 \\
\hline PROSPECT $\ldots \ldots$ & 9,7 & 533 & 398 & 98 & 30 & 68 & 69 \\
\hline PALMER $\ldots \ldots \ldots$ & 8,9 & 533 & 411 & 94 & 24 & 70 & 74 \\
\hline JEWELL $\ldots \ldots \ldots$ & 12,3 & 536 & 428 & 111 & 26 & 85 & 77 \\
\hline DENMAN & & & & & & & \\
\hline $\begin{array}{c}\text { ISLAND } \ldots \ldots \\
\text {. }\end{array}$ & 15,5 & 537 & 437 & 125 & 29 & 96 & 77 \\
\hline $\begin{array}{l}\text { NORTH OF } \\
\text { COURTENAY . }\end{array}$ & 12,3 & 527 & 426 & 119 & 27 & 92 & 77 \\
\hline CHEMULT . . . . & 5,0 & 559 & 384 & 78 & 13 & 65 & 83 \\
\hline SAND POINT I $\ldots$ & 12,7 & 536 & 387 & 127 & 25 & 102 & 80 \\
\hline FERNWOOD ..... & 9,5 & 532 & 386 & 105 & 24 & 81 & 77 \\
\hline CASCADIA $\ldots \ldots$ & 9,7 & 543 & 425 & 102 & 20 & 82 & 80 \\
\hline WIND RIVER. ..... & 9,5 & 530 & 405 & 110 & 23 & 87 & 79 \\
\hline $\begin{array}{l}\text { ANDERTON } \\
\text { ROAD } \ldots \ldots \ldots \ldots\end{array}$ & & & & & & & \\
\hline $\begin{array}{l}\text { ROAD ...... } \\
\text { St-GERMAIN- }\end{array}$ & 14,8 & 543 & 414 & 126 & 27 & 99 & 79 \\
\hline LANGOT $\ldots . .$. & 13,9 & 538 & 431 & 108 & 29 & 79 & 73 \\
\hline CLARKIA ...... & 7,7 & 553 & 403 & 95 & 22 & 73 & 77 \\
\hline LES BARRES G ... & 17,9 & 511 & 396 & 124 & 37 & 87 & 70 \\
\hline LA JONCHẼRE . . & 14,8 & 509 & 413 & 130 & 29 & 101 & 78 \\
\hline LES BARRES L .. & 18,2 & 492 & 407 & 114 & 41 & 73 & 64 \\
\hline NANAIMO $\ldots \ldots$ & 9,4 & 559 & 411 & 118 & 24 & 94 & 80 \\
\hline SAINT-AVIT I $\ldots$. & 20,8 & 521 & 422 & 128 & 38 & 90 & 70 \\
\hline
\end{tabular}




\subsection{Etude de la densité du bois de tige}

\subsection{Corrélation avec le poids anhydre de la partie aérienne}

La figure 3 donne les variations de la densité moyenne des tiges, exprimée en $\mathrm{g} / \mathrm{dm}^{3}$, en fonction du poids anhydre de la partie aérienne, exprimé en $\mathrm{dg}$.

Sur cette figure, et sur toutes les suivantes, les points figuratifs des diverses provenances sont, pour des raisons de commodité, identifiés par une lettre, conformément au tableau d'équivalence ci-dessous :

TABLEAU 2

\begin{tabular}{|c|c|c|c|}
\hline $\begin{array}{l}\text { Lettre } \\
\text { repère }\end{array}$ & Provenance & $\begin{array}{l}\text { Lettre } \\
\text { repère }\end{array}$ & Provenance \\
\hline $\begin{array}{ll}\text { A } & \cdots \\
\text { B } & \cdots \\
\text { C } & \cdots \\
\text { D } & \cdots \\
\text { E } & \cdots \\
\text { F } & \cdots \\
\text { G } & \cdots \\
\text { H } & \cdots \\
\text { J } & \cdots \\
\text { K } & \cdots \\
\text { L } & \cdots \\
M & \cdots\end{array}$ & $\begin{array}{l}\text { PE-ELL } \\
\text { LEWIS } \\
\text { LEABURG I } \\
\text { CAMPBELL RIVER } \\
\text { PEZANIN } \\
\text { SNOQUALMIE PASS } \\
\text { SERANDON } \\
\text { PROSPECT } \\
\text { PALMER } \\
\text { JEWELL } \\
\text { DENMAN ISLAND } \\
\text { NORTH OF } \\
\quad \text { COURTENAY }\end{array}$ & $\begin{array}{ll}\mathrm{N} & \cdots \\
\mathrm{O} & \cdots \\
\mathrm{P} & \cdots \\
\mathrm{Q} & \cdots \\
\mathrm{R} & \cdots \\
\mathrm{S} & \cdots \\
\mathrm{T} & \cdots \\
\mathrm{U} & \cdots \\
\mathrm{V} & \cdots \\
\mathrm{W} & \cdots \\
\mathrm{X} & \cdots \\
\mathrm{Y} & \cdots \\
\mathrm{Z} & \cdots\end{array}$ & $\begin{array}{l}\text { CHEMULT } \\
\text { SAND POINT I } \\
\text { FERNWOOD } \\
\text { CASCADIA } \\
\text { WIND RIVER } \\
\text { ANDERTON ROAD } \\
\text { St-GERMAIN-LANGOT } \\
\text { CLARKIA } \\
\text { LES BARRES G } \\
\text { LA JONCHERE } \\
\text { LES BARRES L } \\
\text { NANAIMO } \\
\text { SAINT-AVIT I }\end{array}$ \\
\hline
\end{tabular}

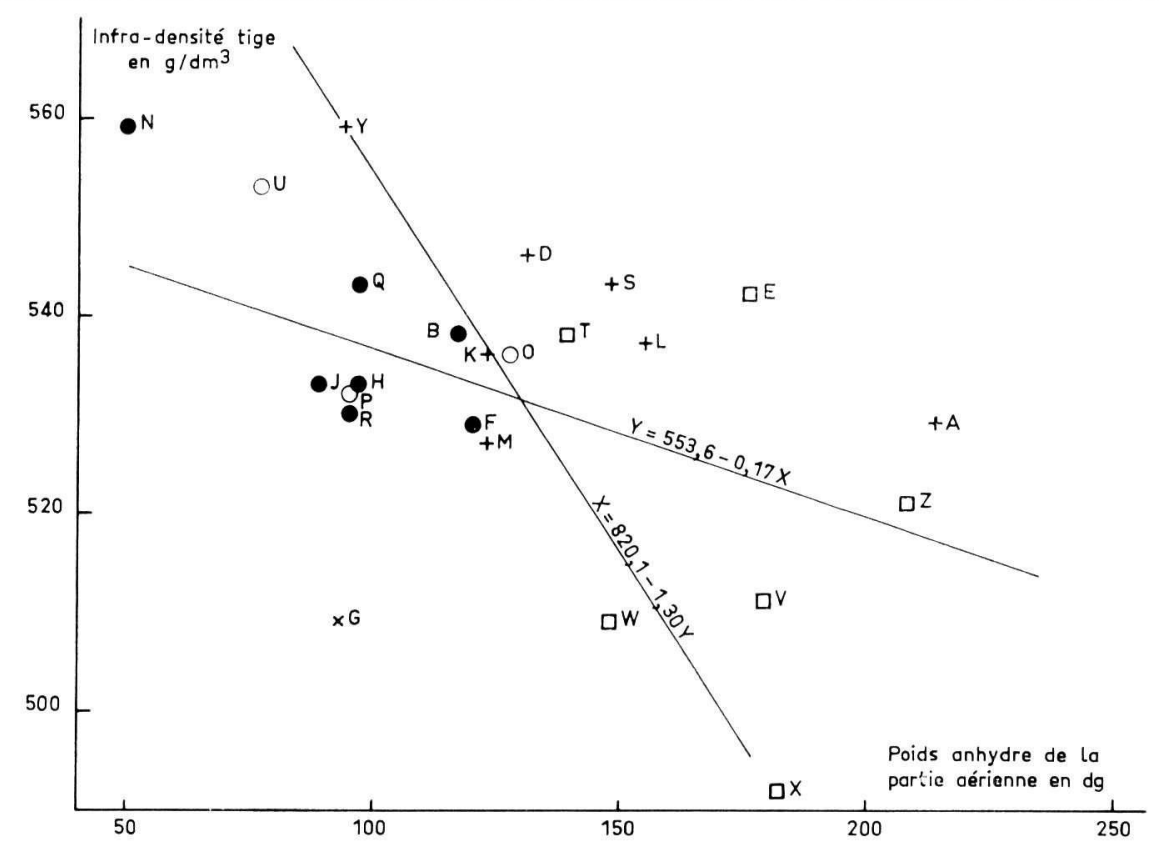

FIG. 3. - Corréiation entre l'infra-densité de la tige et le poids anhydre de la partie aérienne. 
Il existe malheureusement une corrélation négative significative entre ces deux données ( $r=-0,48$ avec 23 degrés de liberté); la formule de la régression de la densité $Y$ exprimée en $\mathrm{g} / \mathrm{dm}^{3}$ en fonction du poids anhydre $X$ exprimée en dg est la suivante :

$$
Y=553,6-0,17 X
$$

Le rapport du coefficient de régression à son écart-type est de $-2,45$, et la diminution de la densité lorsque le poids anhydre de la partie aérienne augmente n'est donc pas imputable au hasard.

Il y a d'ailleurs lieu d'observer que les points figuratifs correspondant à la provenance Serandon (lettre G), qui ne serait pas une provenance d'Abies grandis mais un hybride supposé concolor, et à la provenance Nanaïmo (Y), dont les échantillons provenaient d'une répétition différente, sont parmi ceux qui s'écartent le plus de la droite de régression, et leur élimination, qui serait parfaitement justifiée, ferait donc apparaître une liaison négative encore plus étroite entre densité du bois de tige et poids anhydre de la partie aérienne.

On peut remarquer enfin que les quatre premières provenances au point de vue poids anhydre sont mal classées au point de vue densité : ce sont les provenances Pe-ell $\left(1^{\mathrm{re}}\right.$ et $\left.17^{\mathrm{e}}\right)$, Saint-Avit I ( $2^{\mathrm{e}}$ et $\left.20^{\mathrm{e}}\right)$, Les Barres L ( $3^{\mathrm{e}}$ et $\left.24^{\mathrm{e}}\right)$, Les Barres G $\left(4^{\mathrm{e}}\right.$ et $\left.21^{\mathrm{e}}\right)$; inversement, la provenance Chemult, qui est dernière au point de vue croissance, est première au point de vue densité ; ceci tend à montrer que le poids anhydre de la partie aérienne dépend beaucoup plus du volume apparent que de la densité.

Cependant, quelques provenances paraissent intéressantes comme Pezanin (5e et $\left.7^{\mathrm{e}}\right)$, Denman Island $\left(6^{\mathrm{e}}\right.$ et $\left.10^{\mathrm{e}}\right)$, Anderton Road ( $7^{\mathrm{e}}$ et $\left.5^{\mathrm{e}}\right)$, Saint-Germain-Langot $\left(9^{\mathrm{e}}\right.$ et $\left.8^{\mathrm{e}}\right)$. D'une façon générale, les provenances artificielles, à l'exception de SaintGermain-Langot et Pezanin, sont caractérisées malencontreusement à la fois par une forte vigueur de croissance et une faible densité du bois. Les provenances cascadiennes, par contre, très groupées sur la figure, fournissent du bois dense, mais à croissance relativement lente.

\subsection{Corrélation avec la largeur du cerne 1964 (fig. 4)}

Le coefficient de corrélation est de $-0,37$, c'est-à-dire qu'il n'est pas significatif, mais très voisin du seuil de signification de $5 \%$; cette liaison négative confirme dans une certaine mesure celle de même signe dont il est question au paragraphe précédent; elle n'est pas dépourvue d'intérêt, car le poids anhydre de la partie aérienne intègre un assez grand nombre de facteurs différents : croissance en hauteur, croissance en diamètre, coefficient de forme, densité du bois, volume et densité des aiguilles, alors que le critère maintenant examiné ne correspond qu'à la seule croissance en diamètre durant la dernière année précédant l'arrachage des plants.

\section{3. - Etude de la densité du bois de branche}

\subsection{Corrélation avec la densité du bois de tige (fig. 5)}

La liaison entre ces deux caractéristiques a été étudiée afin de voir si elle pourrait éventuellement être assez forte pour fournir un test non destructif précoce d'appréciation indirecte de la densité tige; il n'en est malheureusement rien, ainsi que le 


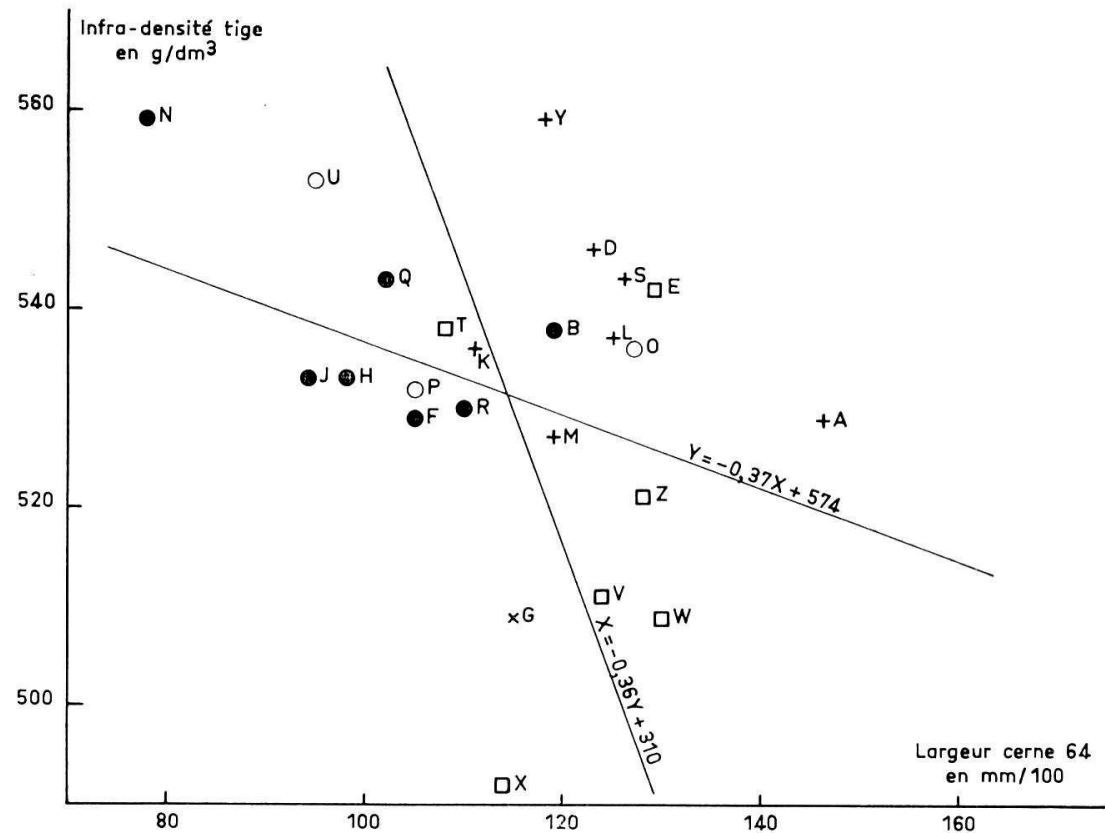

FIG. 4. - Corrélation entre l'infra-densité de la tige et la largeur du cerne 1964.

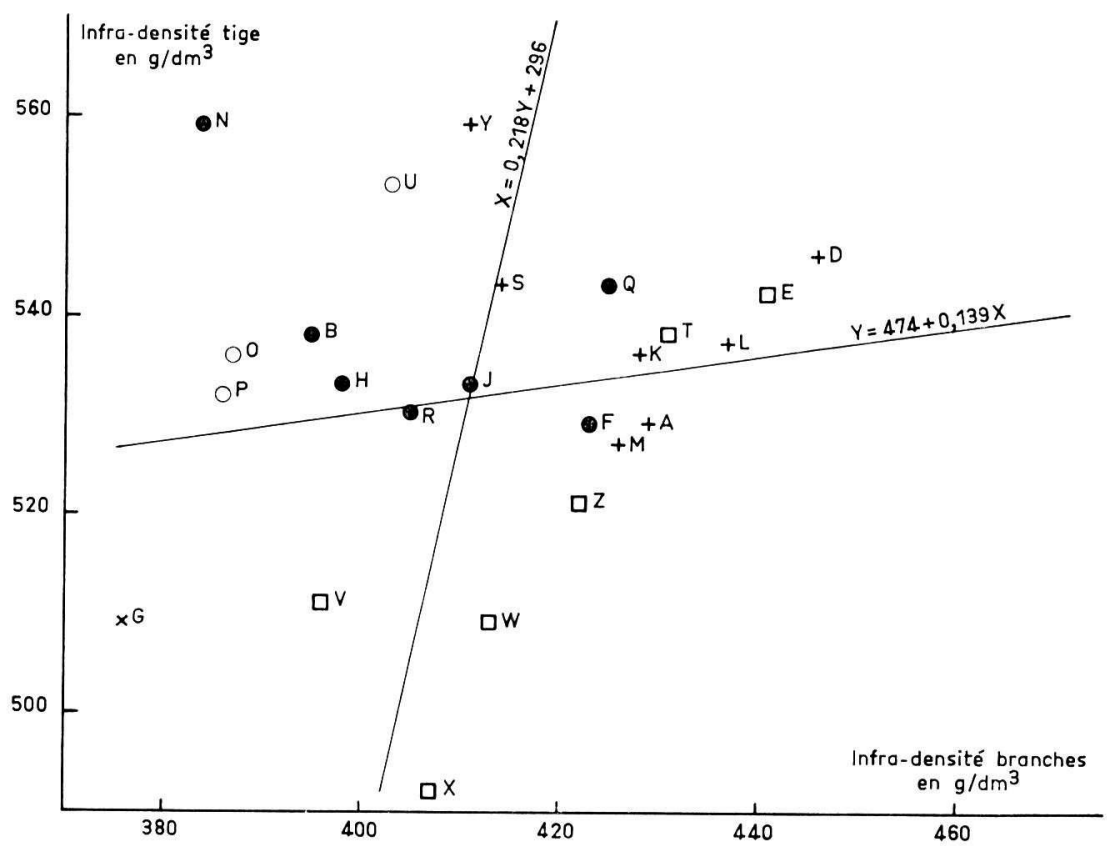

FIG. 5. - Corrélation entre l'infra-densité de la tige et l'infra-densité des branches. 
montre la figure 5 sur laquelle sont reportées les valeurs moyennes des deux variables pour chaque provenance; la dispersion des résultats est considérable; le coefficient de corrélation est cependant positif; il se chiffre à 0,17 , mais il n'est pas significatif, et on peut dire que la liaison est faible au niveau provenance entre la densité du bois de tige et celle du bois de branche.

Au niveau individuel par contre, grâce au nombre élevé de degrés de liberté (470 DL), le coefficient de corrélation, qui est du même ordre $(+0,16)$, devient significatif, mais ceci ne change rien à la conclusion : il n'est pas possible dans le cas particulier de l'Abies grandis à 3 ans d'apprécier, même de façon approximative, la densité $\mathrm{du}$ bois d'une provenance connaissant celle des branches principales $\mathrm{du}$ premier verticille; cependant, cette tendance à une liaison positive entre les deux caractéristiques doit être retenue, car une étude analogue, faite récemment sur le Pin maritime, a conduit à un coefficient de corrélation significatif, et il n'est pas impossible qu'il en soit de même sur Abies grandis lorsqu'on pourra étudier des échantillons plus âgés, ou un nombre de provenances plus important.

\subsection{Corrélation avec le poids anhydre de la partie aérienne}

Contrairement à ce qu'on pouvait supposer, les deux caractéristiques paraissent assez liées au niveau provenance, ainsi que le montre la figure 6 ; le coefficient de corrélation est significatif : $r=+0,48$ avec 23 DL.

La formule de la droite de régression de $Y$ (densité branche en $\mathrm{g} / \mathrm{dm}^{3}$ ) en $X$ (poids anhydre de la partie aérienne en $\mathrm{dg}$ ) est :

$$
Y=383,6+0,22 X
$$

Le rapport du coefficient de régression à son écart-type est de 2,49.

Au total, on retrouve donc une liaison aussi étroite que celle qui existe entre la densité du bois de tige et le poids anhydre de la partie aérienne, mais de sens contraire; on peut penser que la sève élaborée, qui est produite en plus grande abondance dans les plants à fort développement, est utilisée par priorité au niveau des branches, permettant d'obtenir des fibres à parois plus épaisses et, par suite, une densité plus élevée.

\subsection{Corrélation avec la largeur des cernes}

Elle fait l'objet de la figure 7.

Le coefficient trouvé est de 0,38 avec 23 D.L., c'est-à-dire très voisin du seuil de signification de $5 \%$; étant positif, il vient confirmer la liaison trouvée au paragraphe précédent, mais, alors que le poids anhydre de la partie aérienne était fonction de la densité du bois des branches, la largeur du cerne 1964 est bien entendu tout à fait indépendante de ce critère, et le coefficient de corrélation trouvé, bien que moins significatif, n'en est que plus intéressant à noter. 


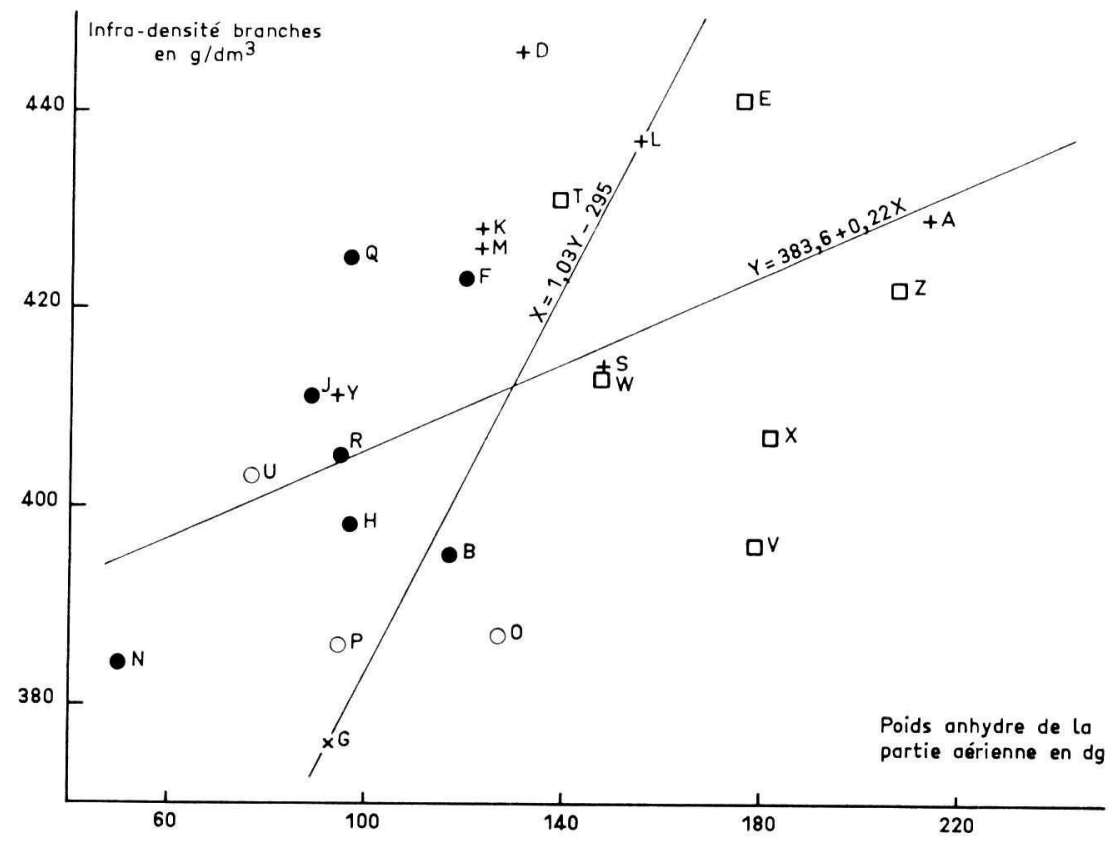

FIG. 6. - Corrélation entre l'infra-densité des branches et le poids anhydre de la partie aérienne.

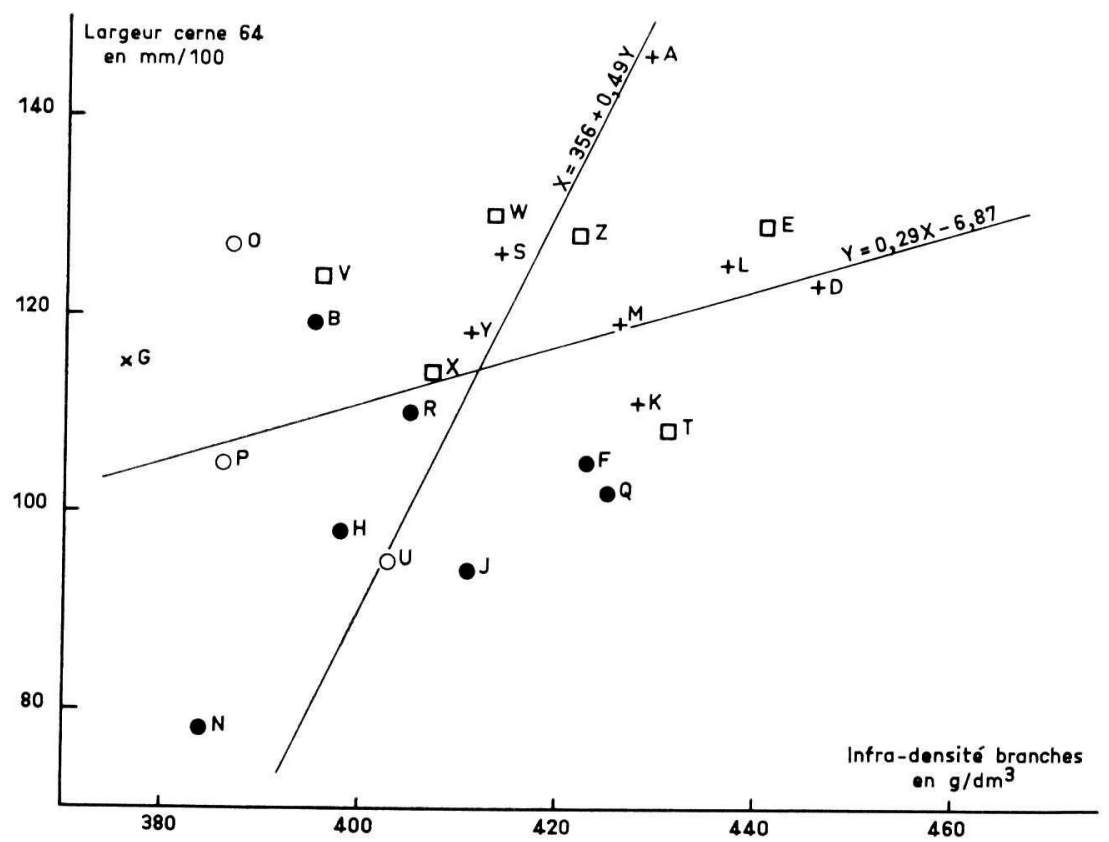

Fig. 7. - Corrélation entre la largeur du cerne 1964 et l'infra-densité des branches. 


\section{4. _ Etude de la structure interne du cerne 1964}

\subsection{Largeur $d u$ cerne}

Outre les corrélations déjà vues aux paragraphes 2.22 et 2.33 , on a étudié celle qui lie la largeur du seul accroissement annuel 1964 au poids anhydre de la partie aérienne; le graphique correspondant fait l'objet de la figure 8.

La liaison est extrêmement étroite : le coefficient de corrélation est de $+0,84$ avec 24 D.L., c'est-à-dire très significatif; la droite de régression donnant la largeur du cerne : $Y($ en $\mathrm{mm} / 100)$ en fonction du poids anhydre de la partie aérienne : $X$ (en $\mathrm{dg}$ ) a pour formule :

$$
Y=74,25+0,30 X
$$

Le rapport du coefficient de régression à son écart-type est de 7,45, c'est-à-dire, lui aussi, très significatif.

Cependant, la hiérarchie des diverses provenances au point de vue rapidité de croissance en diamètre durant la seule dernière année ayant précédé l'arrachage des plants n'est pas exactement la même que pour le poids anhydre total de la partie aérienne ou pour la hauteur moyenne des plants : c'est ainsi que la provenance Pe-ell par exemple prend la tête, très nettement détachée devant Pezanin, alors que Saint-Avit I, première pour la croissance en hauteur, n'est plus que $3^{\mathrm{e}}$; parmi les provenances à faible croissance, Chemult reste dernière, mais est beaucoup plus attardée que pour la croissance en hauteur ; par contre, Serandon, qui était avantdernière d'après ce critère, arrive, au point de vue largeur du cerne 1964, dans une bonne moyenne.

\subsection{Largeur du bois de printemps 1964}

2.421. Corrélation avec le poids anhydre de la partie aérienne

C'est la plus étroite de toutes les liaisons étudiées jusqu'ici, puisque le coefficient de corrélation se chiffre à 0,85 avec $24 \mathrm{DL}$; la formule de la régression de $Y$ (largeur du bois de printemps en $\mathrm{mm} / 100$ ) par rapport à $X$ (poids anhydre de la partie aérienne en $\mathrm{dg}$ ) est :

$$
Y=11,25+0,12 X
$$

avec un rapport du coefficient de régression à son écart-type très considérable de 8,74. Aucune figure n'est cependant fournie pour mettre en évidence cette liaison, le graphique correspondant étant extrêmement voisin du précédent.

2.422. Corrélation avec le pourcentage de plants n'ayant pas achevé leur première vague de croissance en hauteur le $1^{\mathrm{er}}$ juillet 1964

La variabilité au point de vue époque de débourrement étant faible, on pouvait penser que la largeur du bois de printemps serait liée à l'époque à laquelle cesse l'élongation de la tige; on a donc comparé la largeur du bois de printemps 1964 à la seule donnée disponible au point de vue élongation : le pourcentage de plants n'ayant pas cessé leur croissance en longueur le $1^{\text {er }}$ juillet 1964.

La figure 9 donne les résultats de cette confrontation; le coefficient de corrélation, après transformation, est très significatif et s'élève à $+0,60$ avec $24 \mathrm{DL}$; 


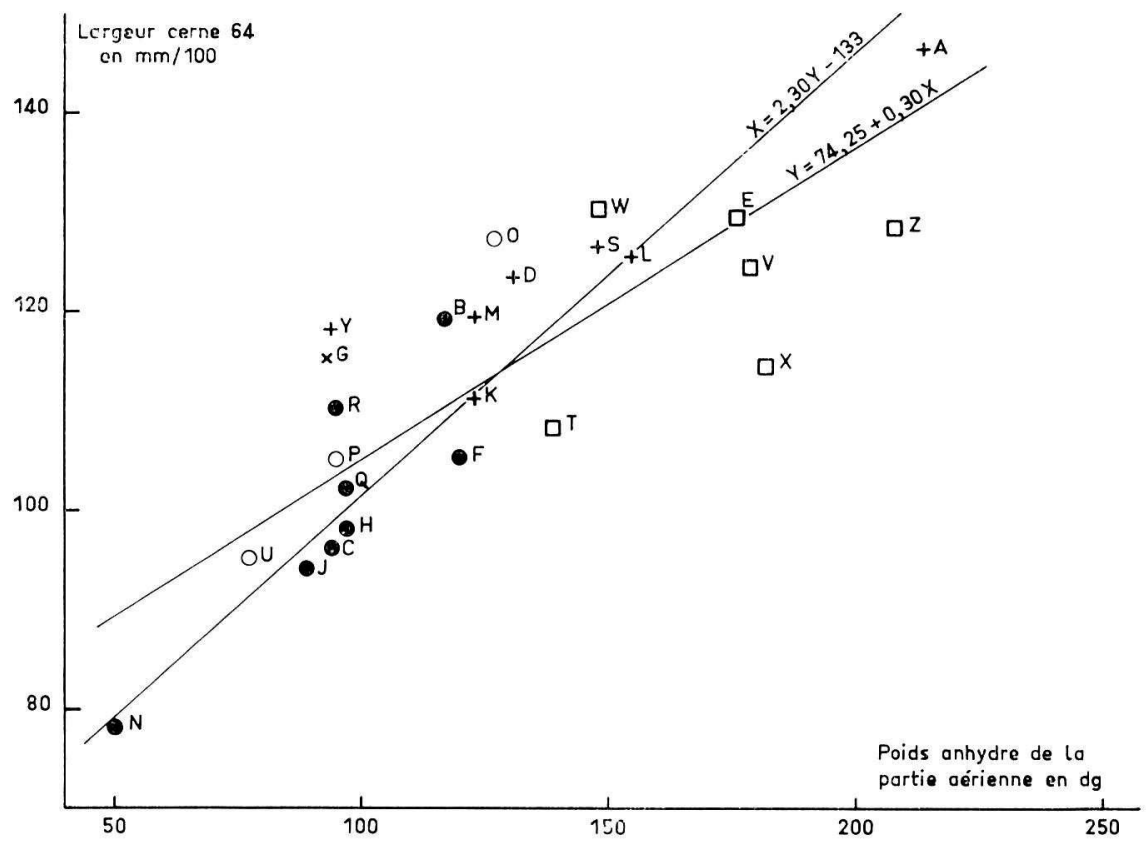

Fig. 8. - Corrélation entre la largeur du cerne 1964 et le poids anhydre de la partie aérienne.

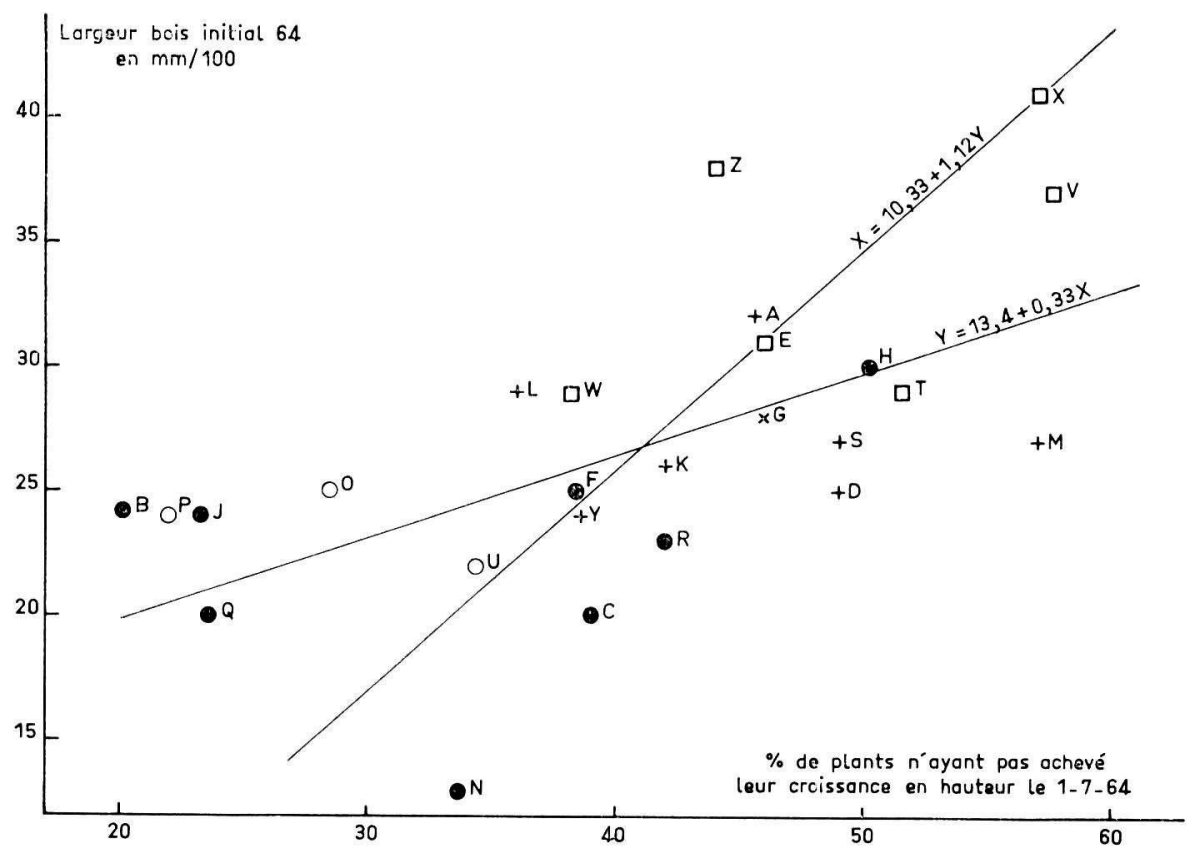

FIG. 9. - Corrélation entre la largeur du bois initial 1964 et le pourcentage de plants n'ayant pas achevé leur croissance en hauteur le 1-7-1964. 
la formule de la droite de régression donnant la largeur du bois de printemps en $\mathrm{mm} / 100: Y$ en fonction de $X(\arcsin \sqrt{\%})$ est :

$$
Y=5,45+0,55 X
$$

Le rapport du coefficient de régression à son écart-type se chiffre à 3,33 montrant que l'augmentation de la largeur du bois de printemps quand s'accroît le pourcentage de plants n'ayant pas cessé leur croissance en longueur le $1^{\text {er }}$ juillet n'est pas l'effet du hasard.

(Il y a lieu d'observer que les abscisses du graphique sont des pourcentages bruts sans transformation, et les droites de régression qui y figurent sont, pour cette raison, quelque peu différentes de celles que l'on obtient sur les données transformées).

Quoi qu'il en soit, la liaison étroite trouvée ici est extrêmement intéressante car elle confirme, au niveau provenance, la théorie suivant laquelle la production de cellules à large lumen et faible épaisseur de membrane du bois initial s'expliquerait par un meilleur approvisionnement de l'assise génératrice en substances de croissance durant la période de développement des bourgeons; c'est, semble-t-il, la première fois que l'on obtient une preuve statistique de l'influence de la durée de la période d'élongation sur la largeur du bois de printemps au stade semis.

\subsection{Etude du bois d'été}

2.431. Corrélation entre la largeur du bois d'été et le pourcentage de plants ayant fait une pousse d'aô̂t

$\mathrm{Au}$ vu des résultats très encourageants de la tentative précédente, un essai analogue a été fait pour expliquer la largeur du bois d'été par le pourcentage de plants ayant fait une pousse d'août cette année là ; ce travail fait l'objet de la figure 10 ; là encore, la liaison est très forte $(r=+0,57$ pour $24 \mathrm{DL})$ et dépasse tout ce qu'on pouvait attendre eu égard au caractère quelque peu hétérogène des deux données confrontées.

La droite de la régression de $Y$ (largeur du bois d'été en $\mathrm{mm} / 100)$ en $X$ (arc sin $\sqrt{\%}$ de plants ayant fait une pousse d'août) est :

$$
Y=35,7+0,97 X
$$

et le rapport du coefficient de régression à son écart-type 3,3 .

Il semblerait que les pousses d'août, dans les provenances où cette deuxième vague de croissance est fréquente, occasionnent, dans le plan transversal, une production supplémentaire de bois d'été, ce qui ne saurait surprendre car, à cette époque de l'année, la circulation de sève est déjà fortement ralentie et les cellules produites jouent beaucoup plus le rôle de tissus de soutien que de tissus conducteurs.

L'étude simultanée des variations de croissance en hauteur et en circonférence au stade semis demanderait cependant à être reprise, car la grande majorité des radiographies auxquelles on a procédé à l'occasion de ce travail fait apparaître dans le 


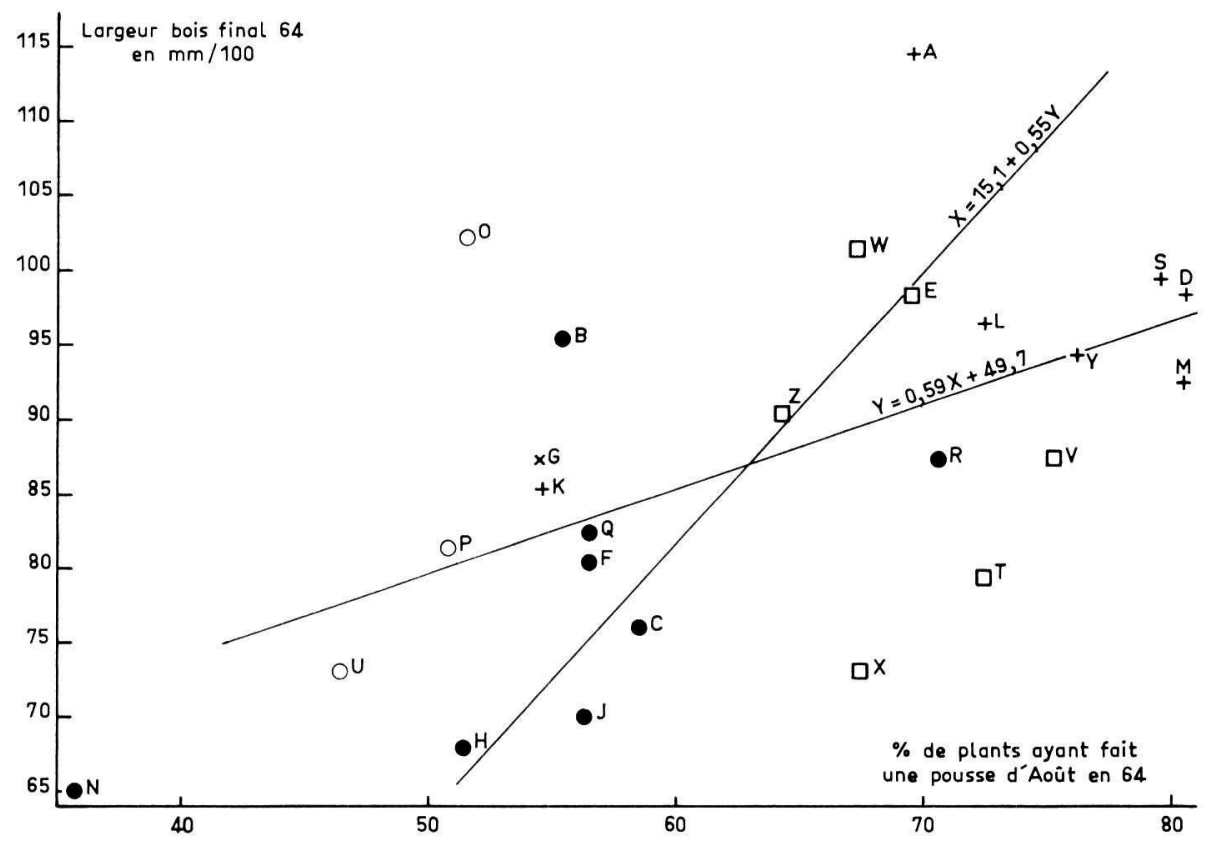

FIG. 10. - Corrélation entre la largeur du bois final 1964 et le pourcentage de plants ayant fait une pousse d'août en 1964.

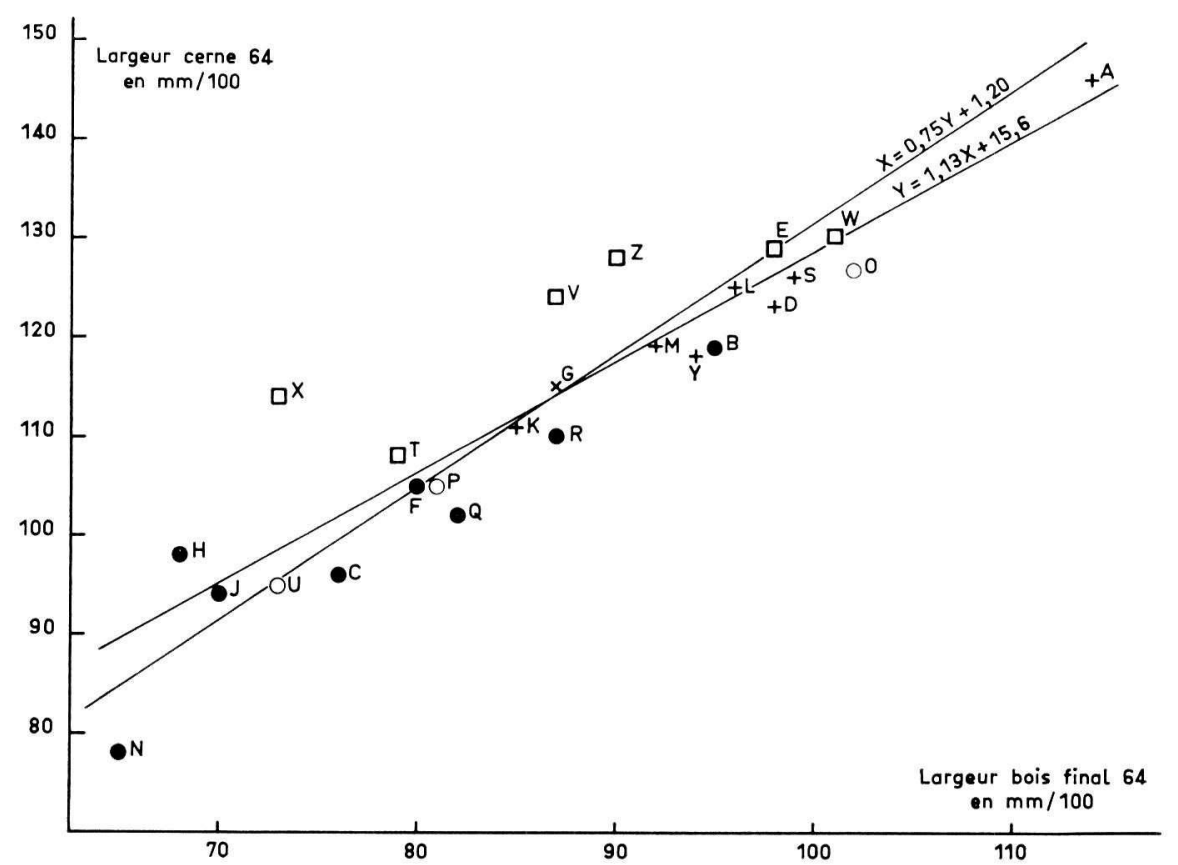

Fig. 11. - Corrélation entre la largeur du cerne 1964 et la largeur du bois final 1964. 
bois d'été une alternance de zones à forte et à faible densité (voir notamment les profils densitométriques des figures 2 et 2 bis), et il serait extrêmement intéressant d'étudier dans quelle mesure ce phénomène peut être lié à des variations éventuelles de la vitesse d'élongation.

\subsection{Corrélation entre la largeur du bois d'été et la largeur totale du cerne}

Mis à part 3 provenances artificielles (Saint-Avit, Les Barres G et Les Barres L), on pourrait croire à une liaison fonctionnelle entre ces deux caractéristiques (fig. 11).

Le coefficient de corrélation est de 0,92 , et la largeur du carne est liée à celle du seul bois d'été par une relation de la forme :

$$
Y=15,6+1,13 X
$$

le rapport du coefficient de régression à son écart-type étant de 11,5.

Ceci tendrait à montrer que l'importance relative de l'accroissement annuel dans les diverses provenances est surtout conditionnée par celle du bois formé pendant la dernière phase de croissance en hauteur, et après l'arrêt de celle-ci, les trois exceptions susmentionnées s'écartant de cette règle par des cernes plus larges à bois final égal, donc par une tendance à produire une proportion plus élevée de bois avant la fin de la période de formation du bois de printemps.

\subsection{Corrélation entre le pourcentage de bois final et l'infra-densité du bois}

La relation étroite qui existe entre la texture (pourcentage de bois final) et la densité d'un même bois est une donnée classique de technologie forestière. Il a cependant paru intéressant de l'étudier ici en raison des conditions très particulières de l'expérience, c'est-à-dire du très jeune âge des échantillons et des difficultés qui en sont résultées dans la détermination de l'une et l'autre des deux caractéristiques en cause.

En fait (fig. 12), on retrouve une liaison significative (coefficient de corrélation $+0,66$ ), ce qui confirme a posteriori dans une certaine mesure la validité des méthodes totalement indépendantes l'une de l'autre qui ont été utilisées pour calculer, aussi bien l'infra-densité que le pourcentage de bois final.

L'équation de la droite de régression de $Y$ (infra-densité en $\mathrm{g} / \mathrm{dm}^{3}$ ) en $X$ (proportion de bois d'été) est :

$$
Y=2,3 X+356
$$

avec un rapport du coefficient de régression à son écart-type de 4,08.

2.434. Classement des diverses provenances au point de vue pourcentage de bois final

Un test de DuCAN a été fait sur cette caractéristique dont l'intérêt est certain, à 


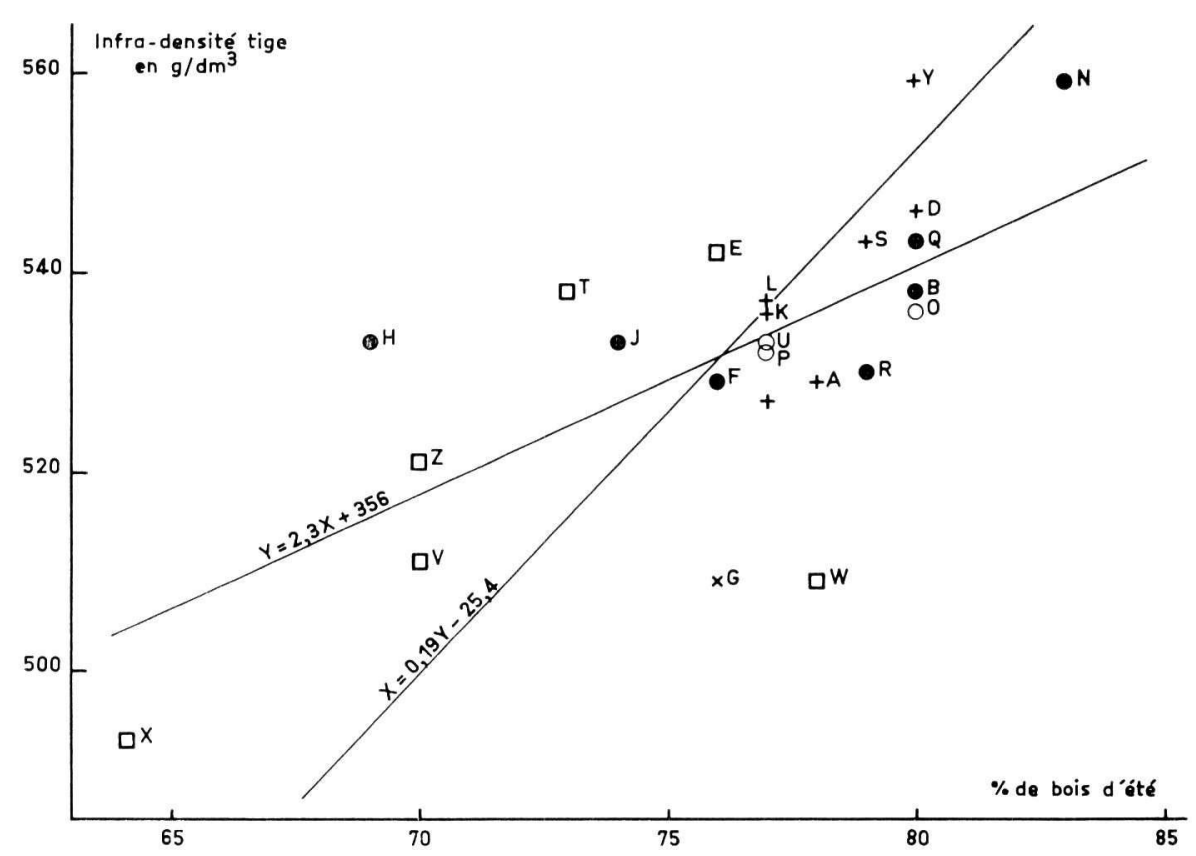

FIG. 12. - Corrélation entre l'infra-densité de la tige et le pourcentage de bois d'été.

la fois au point de vue de la technologie du bois et de la physiologie de l'arbre. Les résultats en sont consignés sur la figure 13 où, comme d'habitude, les provenances qui sont groupées en face d'un même trait ne présentent pas de différence significative entre elles au seuil de $5 \%$ (il convient de noter que, pour ce test, les moyennes indiquées ont été obtenues à partir des pourcentages individuels de bois d'été, alors que, dans le tableau 1, les chiffres de la dernière colonne correspondent à des pourcentages globaux résultant du rapport de la largeur totale de bois final à la largeur totale des cernes pour l'ensemble des échantillons; il y a donc entre ces diverses données les mêmes différences qu'entre un rapport de moyennes et une moyenne de rapports).

On voit que la texture est une donnée qui permet de différencier particulièrement bien les provenances, puisque deux d'entre elles, l'une à fort, et l'autre à faible pourcentage de bois final, sont significativement différentes de l'ensemble des autres; on peut constater de plus que les provenances continentales, bien que n'étant pas toutes à l'intérieur d'une même accolade, sont groupées dans une gamme de texture peu étendue de 75,2 à 79,7\% ; les provenances artificielles au contraire, plus dispersées il est vrai, figurent toutes, à l'exception de La Jonchère, dans le bas du tableau avec des pourcentages de bois final s'échelonnant de 61,8 à $73 \%$; les provenances cascadiennes sont les plus dispersées avec comme extrêmes la provenance Prospect $(68,7 \%)$ et Chemult $(83,2 \%)$. 


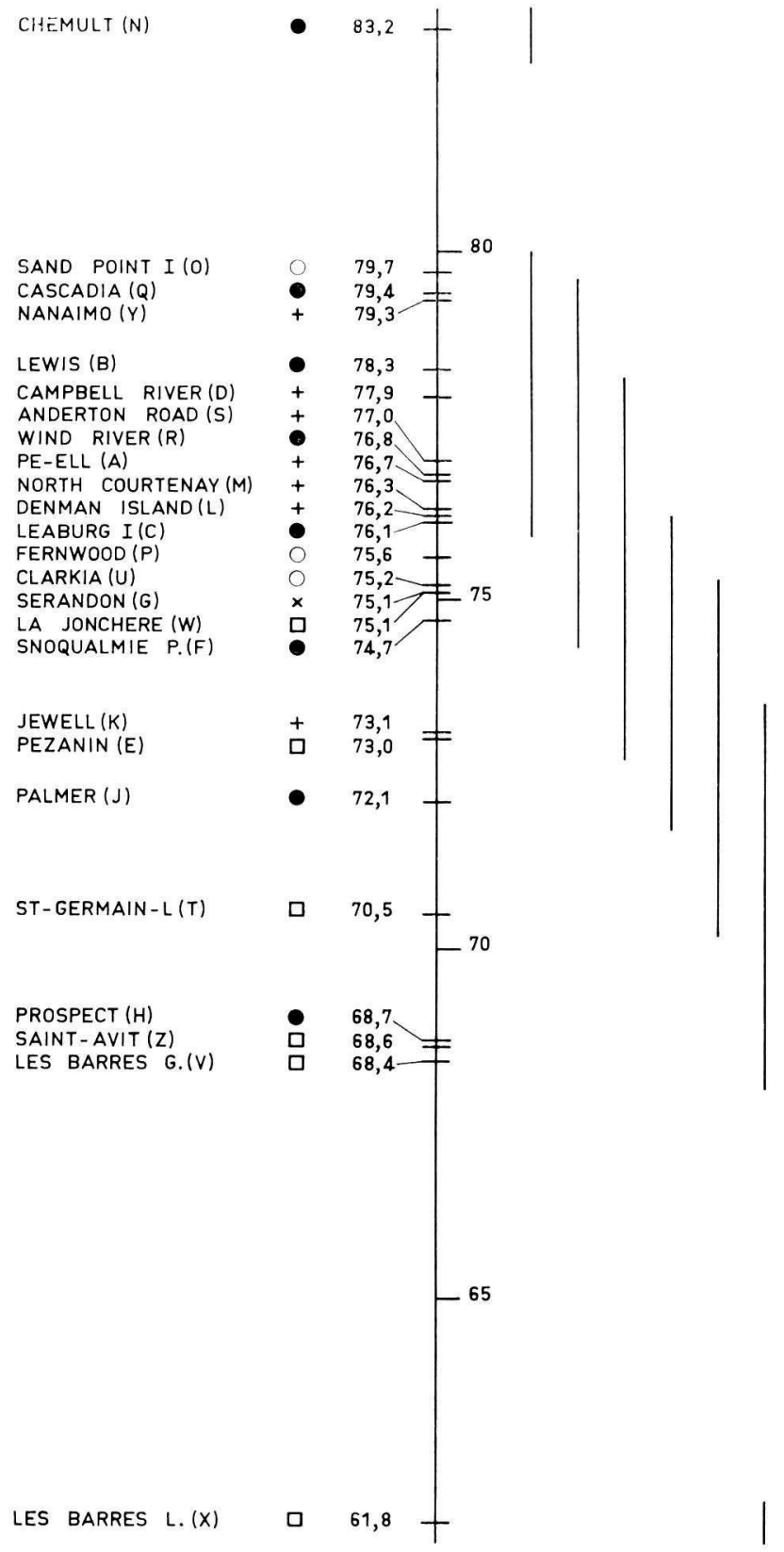

FIG. 13. - Test de comparaison de moyennes sur le pourcentage de bois final. 


\section{CONCLUSION}

Malgré ses imperfections, cette expérience a permis de montrer que des renseignements valables sur la densité du bois, la structure et la composition interne des accroissements annuels pouvaient être obtenus sur des plants de 3 ans dont certains ne dépassaient pas $3 \mathrm{~mm}$ de diamètre au collet.

Bien qu'il y ait en général opposition entre une grande vitesse de croissance et une densité élevée, ou une forte texture du bois, certaines provenances paraissent offrir un compromis satisfaisant entre ces qualités contradictoires : par exemple, Pezanin, ou à un moindre degré Saint-Germain-Langot, pour les provenances artificielles françaises, Pe-Ell, Denman Island ou Anderton Road parmi les provenances côtières américaines.

Certes, on ne peut affirmer que la hiérarchie établie à un âge aussi précoce se maintiendra jusqu'à l'état adulte, tant que l'on n'a pas analysé les corrélations juvénile-adulte pour toutes les provenances en cause; mais une étude récente, consacrée au Pin maritime, et dont les résultats n'ont pas été publiés, a montré que, pour la densité du bois, cette corrélation est très étroite, et il y a peu de chance qu'il en aille différemment pour l'Abies grandis. Au reste, une densité élevée et un fort pourcentage de bois d'été sont, en toute hypothèse, des caractéristiques favorables à l'état juvénile, puisque c'est le bois formé à ce stade qui présente en général les plus graves défauts technologiques.

Outre ces diverses indications relatives au problème de la qualité du bois, des observations intéressantes, et parfois inattendues, ont pu être faites dans des domaines se rattachant à la physiologie de l'arbre : c'est le cas notamment des liaisons positives et significatives qui ont été mises en évidence entre la densité des branches et le poids anhydre de la partie aérienne, entre la largeur du bois initial et le pourcentage de plants ayant poursuivi leur croissance après le $1^{\mathrm{er}}$ juillet, ou entre celle du bois final et la proportion de plants ayant fait des pousses d'août.

De même, la radiographie en couche mince des rondelles de tige a fait apparaître, dans le bois final de presque tous les échantillons, des alternances très marquées de zones à forte et à faible densité; ce phénomène serait à analyser de façon plus approfondie sur le plan anatomique en rapport avec les vitesses relatives de multiplication du méristème apical et du cambium.

D'une façon générale, il est évident que ces études doivent être reprises, en conservant vraisemblablement les mêmes méthodes de travail, mais sur un échantillonnage plus abondant, en analysant d'autres cernes que celui de l'année 1964 où les conditions climatiques ont très bien pu être particulières, et en passant les résultats au crible d'une interprétation statistique plus poussée. Il serait en particulier souhaitable de pouvoir regrouper, dans une analyse des composantes principales unique, facteurs de croissance et critères technologiques; le pourcentage de bois d'été notamment est en effet apparu, au cours de cette première étude, à la fois comme très discriminant et comme peu lié aux diverses caractéristiques auxométriques; on peut par suite penser que la texture du bois pourrait fournir un élément supplémentaire de différenciation et de classement des provenances particulièrement intéressant. 


\title{
SUMMARY
}

\author{
EARLy TESTING OF WOOD QUality ON 25 PROVEnANCES OF Abies grandis
}

In this study have been determined for 25 provenances of Abies grandis, with generally 20 three years old seedlings for each of them, the oven-dried weight of the aerial portion, the wood density of the stem under bark (with a mercury volumenometer) the wood density of the principal branches with bark (by the maximum moisture content method) the ring width and the width of the summerwood (by the way of the radiography) and have been studied the correlations between these various data and certain growth characteristics.

The wood density of the stem is negatively correlated as well with the oven-dried weight of the aerial portion (fig. 3) as with the 1964 ring width (fig. 4), but some french provenances or some coastal american provenances have both a good growth and a satisfactory wood density.

We have found between the wood densities of branches and stems a positive correlation which is significant at the individual level, but not significant at the provenance level (fig. 5) ; so the utilization of branches to provide a non-destructive test of wood quality appreciation is, in these particular conditions, not possible.

On the other hand wood density of branches is correlated significantly with the ovan-dried weight of the aerial portion (fig. 6) and almost significantly with the 1964 ringwidth (fig. 7); both correlations are positive, contrarily to those existing with stem wood density.

The ring width is, of course, in very narrow correlation with the oven-dried weight of the aerial portion (fig. 8), but the grading of provenances is not the same according as the reference is one or the other of both criterions.

Each of the components of the ring 1964 i.e. the earlywood and the laterwood have a significant positive correlation with growth characteristics : the first with the percentage of seedlings having pursued their height growth after the 1st of July (fig. 9), the second with the percentage of seedlings having made an August shoot (fig. 10).

There is an extremely narrow linkage between the ring-width and the width of 1964 summerwood (fig. 11); therefore the summerwood is probably the first responsible of the differences between ringwidths of the different provenances.

The summerwood percentage is highly correlated with the wood density (fig. 12), that is a well known thing in wood technology but supports the accuracy of the methods used for the determination of both characteristics with so small samples.

At last a DUNCAN test (fig. 13) shows that the summerwood percentage is particularly discriminant to differenciate the provenances of Abies grandis which have been studied here.

\section{ZUSAMMENFASSUNG}

\section{Holztechnologische Fruhteste an 25 Provenienzen von Abies grandis (Lindl.)}

An Stichproben von meist 20 Pflanzen pro Herkunft wurden an 25 verschiedenen Provenienzen von Abies grandis Lindl. das Trockengewicht der oberirdischen Pflanzenteile, die Rohdichte des Stammholzes ohne Rinde (Quecksilbervolumenometer), die Rohdichte der Hauptäste ohne Rinde (Methode der integralen Saturation), die Jahrringbreite, sowie die Breite des Spätholzes (Röntgenographie) bestimmt und die allenfalls bestehenden Korrelationen zwischen diesen Merkmalen und den von der «Station d'Amélioration des Arbres Forestiers » (Abteilung für Forstpflanzezüchtung) zur Verfügung gestellten Zuwachsgrössen untersucht.

Zwischen der Rohdichte des Stammholzes und dem Trockengewicht der oberirdischen Pflanzenteile (Abb. 3) als auch der Breite des Jahrringes 1964 (Abb. 4) besteht eine negative Korrelation. Einige französische Herkünfte und auch amerikanische Küstenprovenienzen weisen jedoch ein befriedigendes Wachstum und eine angemessene Rohdichte auf.

Zwischen der Rohdichte des Astholzes und jener des Stammes besteht eine positive Korrelation, welche jedoch nur für die Einzelpflanze nicht aber auf Provenienzebene signifikativ ist (Abb. 5). Die Verwendung von Zweigen zur zerstörungsfreien Prüfung der Holzqualität ist in diesem besonderen Falle daher nicht möglich.

Die Rohdichte des Astholzes ist jedoch signifikant vom Trockengewicht der oberirdischen Pflanzenteile abhängig (Abb. 6) und die Korrelation mit der Jahrringbreite 1964 (Abb. 7) ist nahezu gesichert. Diese beiden Korrelationen sind positiv im Gegensatz zu jenen, die mit der Rohdichte des Stammholzes bestehen. 
Die Jahrringbreite 1964 und das Trockengewicht der oberirdischen Pflanzenteile sind selbstverständlich sehr straff korreliert (Abb. 8), die Klassifizierung der Provenienzen ist jedoch nicht dieselbe für diese beiden Merkmale.

Die zwei Komponenten des Jahrringes 1964, Frühholz und Spätholz, sind positiv und signifikant mit bestimmten Merkmalen des Höhenwachstums der Pflanzen korreliert : die Frühholzbreite mit dem Anteil der Pflanzen welche ihr Höhenwachstum noch nach dem 1. Juli 1964 fortsetzten (Abb. 9) und die Spätholzbreite mit dem Anteil der Pflanzen die einen Johannistrieb ausgebildet hatten (Abb. 10).

Es besteht eine äusserst enge Beziehung auf Provenienzebene zwischen der Spätholzbreite und der Jahrringbreite 1964 (Abb. 11) ; dies scheint zu zeigen, dass die Spätholzbreite einen wesentlichen Anteil an den Zuwachsunterschieden zwischen den Provenienzen zu erklären vermag.

Der Spätholzanteil ist sehr eng mit der Rohdichte des Jahrringes korreliert (Abb. 12). Diese, in der Holztechnologie allgemein bekannte Korrelation, erlaubte in unserem speziellen Fall die Prüfung der angewandten Methoden zur Bestimmung dieser beiden Merkmale bei Frühtesten an sehr kleinen Pflanzen.

Abschliessend konnte durch einen Vergleich der Durchschnitte gezeigt werden, dass der Spätholzanteil zur Unterscheidung der Provenienzen besonders geeignet ist. 